\title{
Stress Assessment by Salivary Biomarkers in Patients with Lung Cancer Undergoing Chemotherapy
}

\author{
Maiko Kitajima $^{1 *}$, Yuka Noto ${ }^{1}$, Umi Kudo ${ }^{1}$, Mayumi Urushizaka ${ }^{1}$, Toshiko Tomisawa ${ }^{1}$, Chieko Itaki $^{1}$, \\ Kumiko Kawasaki ${ }^{1}$, Hideaki Yamabe ${ }^{1}$, Akihito Hayashi ${ }^{2}$, Shingo Takanashi ${ }^{2}$ \\ ${ }^{1}$ Graduate School of Health Sciences, Hirosaki University, Hirosaki, Japan; ${ }^{2}$ Department of Respiratory Medicine, Hirosaki Univer- \\ sity Hospital, Hirosaki, Japan. \\ Email: *kitajima@cc.hirosaki-u.ac.jp
}

Received February $23^{\text {rd }}, 2012$; revised March 20 ${ }^{\text {th }}, 2012$; accepted April $8^{\text {th }}, 2012$

\begin{abstract}
Background: It is important for medical staffs to understand the state of stress of patients with cancer. The authors investigated stress assessment in patients with lung cancer undergoing chemotherapy. Materials and Method: Salivary cortisol and $\alpha$-amylase were measured as stress markers before and after chemotherapy in 10 patients. Emotional status was assessed using the Profile of Mood States (POMS) questionnaire, and Quality of Life (QOL) was assessed using the Functional Assessment of Cancer Therapy-Lung (FACT-L) questionnaire. Results: No significant differences were found between the before and after chemotherapy in salivary levels of cortisol or $\alpha$-amylase, the POMS and QOL scores. The salivary cortisol levels correlated with the POMS subscales of anger-hostility, and confusion. A negative correlation was observed between the salivary cortisol level and the QOL full score. The FACT-L full score correlated with the POMS subscales of depression-dejection, fatigue, and confusion. Conclusions: Measurements of salivary stress markers are noninvasive and may be useful for assessing the stress state of the patients
\end{abstract}

Keywords: Stress; Lung Cancer; Chemotherapy; Cortisol; QOL

\section{Introduction}

Lung cancer is the leading cause of cancer-related deaths in Japan since 1998, and the incidence of this disease is increasing yearly due to smoking and increase of elderly people. Patients who suffer from lung cancer are usually treated with surgery, chemotherapy and/or radiotherapy. Recently, chemotherapy has been shown to be very effective for patients with small cell lung cancer. It is very important for medical staff to administer chemotherapy safely and correctly, so as to ensure optimal outcomes for these patients. It is also important to reduce the level of pain experienced by the patient, maintain the physical condition of the patient, minimize the toxic side-effects of chemotherapy, and reduce physical and emotional stresses.

In 1950, Selye described the General Adaptation Syndrome for the response of the body to stress [1,2]. Lazarus described stress as a process involving complex interactions between the environment and a living body [3]. Patients who have cancer undergo numerous physical and emotional stresses. The stresses suffered by patients who are undergoing chemotherapy for cancer are related to the diagnosis of cancer, notification of malignancy,

*Corresponding author. fear of relapse, symptoms of cancer, changes in life-style, and the toxic side-effects of the chemotherapy. Excessive stress may debilitate the immune system, resulting in complications of cancer and exacerbating the cancer itself. Sandra and colleagues reported that flatter and higher diurnal cortisol levels in salivary slopes predicted shorter subsequent survival time in patients with breast cancer [4], which suggests that the degree of stress influences the prognosis of the cancer patient.

In the present study, the author investigated stress assessment in patients with lung cancer who are receiving chemotherapy by examining biomarkers in the saliva and using the Profile of Mood States (POMS) questionnaire. The author also examined the correlation between two stress markers in the saliva and the Quality of Life (QOL) index.

\section{Materials and Methods}

The present study included 10 patients $(8$ men and 2 women) who received chemotherapy for lung cancer at Hirosaki University Hospital. This study was approved by the Committee for Medical Ethics of Hirosaki University, and the patients gave informed consent. Each subject had a Performance Status (PS) of 0 - 2, and none 
of the subjects were receiving hormone therapy. A PS of 0 - 2 means that the subject can sit up for more than 6 hours during daytime. The average age was 65.8 years \pm 8.5 (range, 50 - 76 years). The patients had been diagnosed as stage $3 A(n=3)$, stage $3 B(n=2)$ or stage $4(n=5)$.

The subjects were required to abstain from eating and drinking anything except water and from brushing their teeth $1 \mathrm{~h}$ before the experiments, which were performed in the period between 14:00 $\mathrm{h}$ and 18:00 $\mathrm{h}$, to minimize any circadian rhythm effects.

Emotional status was assessed using the Japanese version of POMS, and QOL was assessed using the Functional Assessment of Cancer Therapy-Lung (FACT-L). The participants completed these questionnaires after the collection of salivary samples, which was performed once a week (Figure 1).

\subsection{Assaying of Biomarkers in the Saliva of Patients with Lung Cancer}

Salivary samples were obtained from the patients using Salivette (Sarstedt AG \& Co., Nümbrecht, Germany). Saliva was easily collected by allowing the subject to chew on a roll-shaped saliva collector after initially swallowing the saliva present in the mouth. The samples were centrifuged for 5 minutes at $3000 \mathrm{rpm}$, and the supernatants were frozen at $-80^{\circ} \mathrm{C}$ until use. Salivary samples collected for about 2 weeks were analyzed for the levels of the biomarkers cortisol and $\alpha$-amylase. Salivary cortisol was estimated using a salivary cortisol enzyme immunoassay kit (Salimetrics, State College, PA, USA), and salivary $\alpha$-amylase was estimated using the Phadebas Amylase Test (Magle Life Sciences, Lund, Sweden). Alpha-amylase (Sigma Chemical Co., St. Louis, MO, USA) was used as the standard.

\subsection{The Stress Evaluation Index}

Evaluation of emotional status was based on subjective assessment tools, such as the POMS questionnaire and the State-Trait Anxiety Inventory (STAI) score. The POMS questionnaire, which was developed and validated

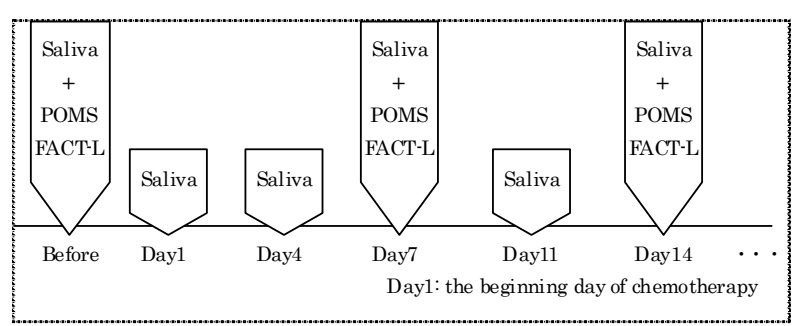

Figure 1. Protocol of research. Saliva was obtained before chemotherapy, day 1, 4, 7, 11 and 14 from the beginning of chemotherapy. POMS and FACT-L were performed before chemotherapy, day 7 and day 14 from the beginning of chemotherapy. by McNair et al. in the US [5], is a self-administered instrument that consists of 65 items designed to identify and assess transient mood states. It measures six mood or affective states: 1) "Tension-Anxiety"; 2) "DepressionDejection"; 3) "Anger-Hostility"; 4) "Vigor"; 5) "Fatigue"; and 6) "Confusion". Many researchers have used this tool in studies of patients with various diseases, including cancer, AIDS, and multiple sclerosis.

Yokoyama translated the POMS questionnaire into Japanese and validated it [6]. The shortened version of the POMS questionnaire consists of 30 items and can be completed in only 10 minutes. It is possible to measure the changes in feelings before and after an intervention. The shortened version generates the same results as the original POMS questionnaire. A standardization score is computed from the measurements and is compared with the average according to age.

\subsection{QOL of Patients with Lung Cancer}

In recent years, there have been discussions regarding the definition of QOL and the methodologies used for assessing QOL in cancer patients. QOL is significantly affected by the occurrence of side-effects, such as nausea/vomiting, loss of appetite, and diarrhea [7]. However, the relationship between the degree of stress and QOL has not yet precisely defined.

The Functional Assessment of Cancer Therapy-General (FACT-G) scale was developed and validated by Cella et al. [8]. In Japan, Fumimoto et al. [9] demonstrated the clinical validity of the cross-cultural validation of the Japanese FACT-G scale. FACT-G questionnaires consist of four domains: 1) Physical Well-Being (PWB); 2) Social/Family Well-Being (SWB); 3) Emotional Well-Being (EWB); and 4) Functional Well-Being (FWB). For patients with lung cancer, the FACT-L questionnaire is used in combination with the Lung Cancer Subscale (LCS). The FACT-L (version 3) questionnaire is a 44-item self-reporting instrument that measures a multidimensional QOL $[10,11]$.

\subsection{Statistical Analysis}

All data are expressed as the mean \pm standard deviation and analyzed using the SPSS 11.5 software. Results were compared using repeated measure ANOVA and Pearson's rank correlation test. $P<0.05$ was considered to be statistically significant.

\section{Results}

\subsection{Biomarkers in the Saliva of Patients with Lung Cancer Undergoing Chemotherapy}

Saliva samples were collected $7.5 \pm 2.7$ times. The mean baseline cortisol level for the patients was $0.015-2.14$ 
$\mu \mathrm{g} / \mathrm{dl}$, and the mean $\alpha$-amylase level was $4.2-522.2$ $\mathrm{U} / \mathrm{ml}$ (Table 1). There were no significant differences among the before and during chemotherapy in the salivary levels of cortisol and $\alpha$-amylase.

\subsection{POMS and FACT-L Scores for Patients with Lung Cancer Undergoing Chemotherapy}

The POMS scores for the patients are presented in Table 2. Both salivary samples and questionnaires were collected 2 to 4 times (total of 26 times). No significant differences were found between the pre-chemotherapy and the 7 th day of chemotherapy in the POMS questionnaire scores. Similarly, there were no significant differences between the pre-chemotherapy and the 7th day of chemotherapy in the scores on the QOL questionnaires.

\subsection{Relationship between Biomarkers in the Saliva and the POMS Scores}

The salivary cortisol levels showed correlations with the POMS subscales of anger-hostility $(\mathrm{r}=0.419, P<0.05)$, and confusion $(\mathrm{r}=0.441, P<0.05)$ (Table 3). No significant relationship was noted between the salivary $\alpha$-amylase levels and the POMS scores.

\subsection{Relationship between Salivary Biomarkers or POMS and QOL}

A negative correlation was observed between the salivary cortisol levels and the QOL full score $(\mathrm{r}=-0.590, P<$ 0.01 ), which means that the higher the salivary cortisol levels, the lower the QOL full score (Figure 2).

The FACT-L and POMS questionnaires were administered a total of 24 times. The FACT-L full score significantly correlated with the POMS subscales of depression-dejection $(\mathrm{r}=-0.656, P<0.01)$, anger-hostility $(\mathrm{r}=$ $-0.481, P<0.05)$, fatigue $(\mathrm{r}=-0.560, P<0.01)$, and

Table 1. Progress of salivary biomarkers among before and during chemotherapy.

\begin{tabular}{ccc}
\hline & Amylase $(\mathrm{U} / \mathrm{ml})$ & Cortisol $(\mu \mathrm{g} / \mathrm{dl})$ \\
\hline Before & $119.62 \pm 160.00$ & $0.33 \pm 0.59$ \\
Day 1 & $59.10 \pm 74.28$ & $0.42 \pm 0.38$ \\
Day 4 & $74.28 \pm 82.76$ & $0.39 \pm 0.25$ \\
Day 7 & $45.63 \pm 42.10$ & $0.47 \pm 0.53$ \\
Day 8 & $69.96 \pm 55.88$ & $0.68 \pm 0.59$ \\
Day 11 & $84.09 \pm 40.89$ & $0.46 \pm 0.34$ \\
Day 14 & $56.67 \pm 32.56$ & $0.36 \pm 0.38$ \\
& & $($ Mean \pm SD) \\
& & non-significant \\
\hline
\end{tabular}

Table 2. POMS test score $(n=10)$. No difference was there between before and after chemotherapy.

\begin{tabular}{ccc}
\hline ScorelDay & $\begin{array}{c}\text { Before chemotherapy } \\
\text { Mean } \pm \text { SD }\end{array}$ & $\begin{array}{c}\text { After chemotherapy } \\
\text { Mean } \pm \text { SD }\end{array}$ \\
\hline POMS subscale & & \\
Tension-Anxiety & $48.7 \pm 12.8$ & $47.2 \pm 9.5$ \\
Depression-Dejection & $50.0 \pm 13.0$ & $53.1 \pm 11.1$ \\
Anger-Hostility & $42.8 \pm 4.9$ & $44.8 \pm 7.9$ \\
Vigor & $48.8 \pm 13.1$ & $49.1 \pm 13.3$ \\
Fatigue & $46.7 \pm 7.1$ & $50.9 \pm 12.4$ \\
Confusion & $50.0 \pm 12.2$ & $50.3 \pm 13.8$ \\
\hline
\end{tabular}

Table 3. Relationship between salivary cortisol levels and POMS score in patients with lung cancer $(n=26)$. The salivary cortisol levels showed positive correlations with the POMS subscales of anger-hostility and confusion.

\begin{tabular}{ccc}
\hline & & $\begin{array}{c}\text { Pearson's correlation } \\
\text { coefficient }\end{array}$ \\
\hline & Tension-Anxiety & 0.147 \\
POMS & Anger-Hostility & 0.338 \\
& Vigor & $0.419^{*}$ \\
& Fatigue & -0.167 \\
& Confusion & 0.246 \\
\end{tabular}

$* p<0.05$

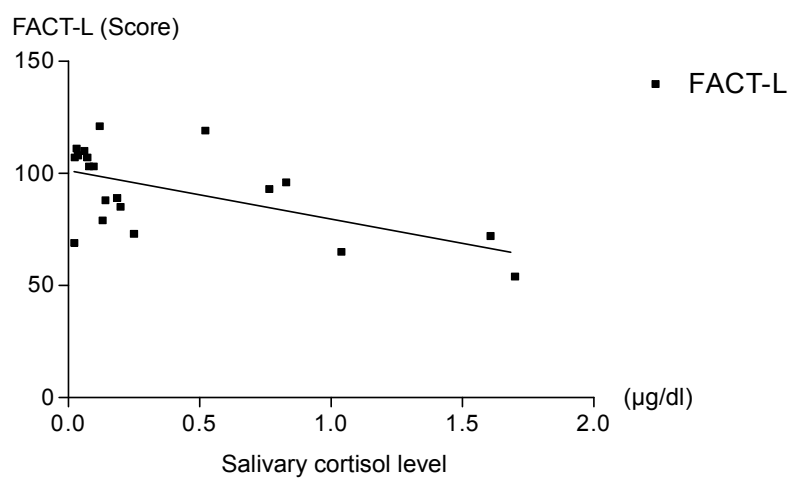

Figure 2. Correlation of the salivary cortisol levels and FACT-L score $(n=24)$. A negative correlation was observed between the salivary cortisolevels and the QOL full score $(\mathrm{r}=-0.590, P<0.01)$.

confusion $(\mathrm{r}=-0.589, P<0.01)$ (Table 4). There was no significant correlation between the salivary $\alpha$-amylase levels and POMS scores.

\section{Discussion}

Patients with cancer who are receiving chemotherapy are 
Table 4. Relationship between POMS and FACT-L in patients with lung cancer $(n=24)$. The FACT-L full score significantly correlated with the POMS subscales of depression-dejection, anger-hostility, fatigue, and confusion.

\begin{tabular}{|c|c|c|c|c|c|c|c|}
\hline & & \multicolumn{6}{|c|}{ FACT-L } \\
\hline & & Full score & PWB & SWB & EWB & FWB & LCS \\
\hline \multirow{7}{*}{ POMS } & Tension-Anxiety & -0.290 & $-0.561 * *$ & 0.089 & $-0.510^{* *}$ & -0.141 & 0.008 \\
\hline & Depression-Dejection & $-0.656 * *$ & $-0.745^{* *}$ & -0.071 & $-0.526 * *$ & $-0.481^{*}$ & $-0.422 *$ \\
\hline & Anger-Hostility & $-0.481^{*}$ & $-0.713 * *$ & 0.122 & $-0.632 * *$ & -0.371 & -0.123 \\
\hline & Vigor & 0.245 & 0.251 & -0.241 & $0.691 * *$ & 0.326 & -0.229 \\
\hline & Fatigue & $-0.560 * *$ & $-0.560 * *$ & -0.090 & $-0.495 *$ & $-0.492 *$ & -0.191 \\
\hline & Confusion & $-0.589 * *$ & $-0.649 * *$ & 0.008 & $-0.644 * *$ & $-0.427^{*}$ & -0.320 \\
\hline & \multicolumn{3}{|c|}{ Pearson's correlation coefficient } & \multicolumn{4}{|c|}{$* \mathrm{p}<0.05 * * \mathrm{p}<0.01$} \\
\hline
\end{tabular}

PWB: Physical well-being; SWB: Social/family well-being; EWB: Emotion well-being; FWB: Functional well-being; LCS: Lung cancer subscale.

usually exposed to various kinds of stresses. Excessive stress may worsen the physical and mental conditions of these patients and exacerbate the cancer itself. In the present study, the author investigated stress assessment in patients with lung cancer who were receiving chemotherapy, by assaying biomarkers in the saliva and administering the POMS questionnaire, thereby examining the correlations between the levels of two stress markers in the saliva and the QOL index.

Numerous physiologic measures have been evaluated for their roles in the stress response, e.g., pulse rate, blood pressure, sweating, and the cortisol, epinephrine, and norepinephrine levels [12]. One of the most common objective measures of stress involves the assaying of neuroendocrine markers. It is well known that the cortisol level in the blood increases under mental stress or pain [12]. It has also been reported that the salivary cortisol level increases with psychological stress [12-14], and correlates with the level of serum cortisol [14]. The levels of salivary $\alpha$-amylase are associated with changes in the blood during exercise and psychosocial stress [14]. Recently, it has been shown that the concentration of $\alpha$-amylase increases after exposure to acute mental stress [15]. Since salivary measurements are non-invasive, salivary biomarkers may be useful clinical indicators of mental stress.

The levels of $\alpha$-amylase and cortisol in the saliva of patients were measured before and during chemotherapy in the present study. No significant differences were found among the before and during chemotherapy in the salivary levels of cortisol and $\alpha$-amylase.

Kajdaniuk et al. reported increased cortisol levels, which might be the result of stress, in the blood samples of women with breast cancer before the first cycle of supplementary chemotherapy [16]. A similar result was expected for patients with lung cancer. However, the salivary biomarkers did not indicate a state of stress in these patients.

In the present study, there were no significant differences between the before and after chemotherapy in the scores on the POMS and QOL questionnaires. However, the salivary cortisol levels correlated with the POMS subscales of anger-hostility, and confusion, whereas the salivary $\alpha$-amylase levels and POMS scores showed no significant relationship. A negative correlation was observed between the salivary cortisol levels and QOL full scores, which means that the higher the level of salivary cortisol, the lower is the full score for QOL. The FACT-L full score was significantly correlated with the POMS subscales of depression-dejection, anger-hostility, fatigue, and confusion. The salivary level of cortisol may reveal significant associations with several psychological characteristics.

Therefore, cortisol in saliva may serve as a negative profile marker in patients with cancer. Stress factors include age, sleeping quality, gender. However, no agerelated differences in salivary cortisol levels were observed between the groups. Cortisol levels have been associated with significantly poorer sleep quality [17]. However, all of the participants in the present study reported high-quality sleep. In addition, patients with lung cancer who are receiving chemotherapy sometimes worry about the side-effects of chemotherapy, such as nausea, and hair loss. The present study revealed that the higher the level of salivary cortisol, the lower was the full score for QOL. Therefore, it appears that low-level physical activity and the appearance of symptoms related to lung cancer may cause high levels of stress.

No significant correlation was observed between the $\alpha$-amylase levels and QOL index, possibly because the salivary level of $\alpha$-amylase reflects a high-level reaction to temporary or acute stress.

"Anger-Hostility" denotes bad-tempered and a high level of nervousness. "Confusion" means distraught. Be- 
ing placed in a hospital environment for first-time chemotherapy, the patients have a high risk of suffering from confusion. The mean salivary cortisol levels have been reported to correlate negatively with the subscales of appraisal, belonging, and tangible social support in the Interpersonal Support Evaluation List [18]. Therefore, mental health support is essential to maintain good QOL during the total period of chemotherapy.

It is very important for medical staff to understand the state of stress of patients with cancer and to support these patients. Measurements of stress markers in saliva are noninvasive and may be useful for assessing clinically the mental stress status of a patient.

\section{Acknowledgements}

The main support for this study was provided by the medical staff of Hirosaki University Hospital, and I would like to thank all the participants, especially those patients who devoted so much time and energy to this study.

\section{REFERENCES}

[1] H. Selye and C. Fortier, "Adaptive Reaction to Stress," Psychosomatic Medicine, Vol. 12, 1950, pp. 149-157.

[2] H. Selye, "Stress and the General Adaptation Syndrome," British Medical Journal, Vol. 1, 1950, pp. 1383-1392. doi:10.1136/bmj.1.4667.1383

[3] R. S. Lazarus and S. Folkman, "Stress, Appraisal, and Coping," Springer Pub, New York, 1984.

[4] S. E. Sephton, R. M. Sapolsky, H. C. Kraemer and D. Spiegel, "Diurnal Cortisol Rhythm as a Predictor of Breast Cancer Survival," Journal of the National Cancer Institute, Vol. 92, No. 12, 2000, pp. 994-1000. doi:10.1093/jnci/92.12.994

[5] D. M. McNair, M. Lorr and L. F. Droppleman, "Profile of Mood States," Educational and Industrial Testing Service, San Diego, 1992.

[6] K. Yokoyama, S. Araki, J. Osuga, T. Karita, M. Kurokawa and K. Koda, "Production of the Japanese Edition of Profile of Mood States (POMS): Assessment of Reliability and Validity," Japanese Journal of Public Health, Vol. 37, 1990, pp. 913-918 (In Japanese).

[7] S. Morita, K. Kobayashi, K. Eguchi, T. Matsumoto, M. Shibuya, Y. Yamaji, J. Sakamoto and Y. Ohashi, "Influence of Clinical Parameters on Quality of Life During Chemotherapy in Patients with Advanced Non-Small Cell Lung Cancer: Application of a General Linear Model," Japanese Journal of Clinical Oncology, Vol. 33, No. 9, 2003, pp. 470-476. doi:10.1093/jjco/hyg083

[8] D. F. Cella, D. S. Tulsky, G. Gray, B. Sarafian, E. Linn, A. Bonomi, M. Silberman, S. B. Yellen, P. Winicour, J. Brannon and F. David, "The Functional Assessment of
Cancer Therapy Scale Development and Validation of the General Measure," Journal of Clinical Oncology, Vol. 11, No. 3, 1993, pp. 570-579.

[9] A. Yoshimura, K. Kobayashi, H. Fumimoto, Y. Fujiki, S. Eremenco and S. H. Kudoh, "Cross-Cultural Validation of an International Questionnaire, the General Measure of the Functional Assessment of Cancer Therapy Scale (FACT-G), for Japanese," Quality of Life Research, Vol. 10, No. 8, 2001, pp. 701-709. doi:10.1023/A:1013851216181

[10] D. F. Cella, A. E. Bonomi, S. R. Lloyd, D. S. Tulsky, E. Kaplan and P. Bonomi, "Reliability and Validity of the Functional Assessment of Cancer Therapy-Lung (FACTL) Quality of Life Instrument," Lung Cancer, Vol. 12, No. 3, 1995, pp. 199-220. doi:10.1016/0169-5002(95)00450-F

[11] E. Saitoh, Y. Yokomizo, C. H. Chang, S. Eremenco, H. Kaneko and K. Kobayashi, "Cross-Cultural Validation of the Japanese Version of the Lung Cancer Subscale on the Functional Assessment of Cancer Therapy-Lung," Journal of Nippon Medical School, Vol. 74, No. 6, 2007, pp. 402-408. doi:10.1272/jnms.74.402

[12] K. M. Hargreaves, "Neuroendocrine Markers of Stress," Anesthesia Progress, Vol. 37, 1990, pp. 99-105.

[13] M. Biondi and A. Picardi, "Psychological Stress and Neuroendocrine Function in Humans: The Last Two Decades of Research," Psychotherapy and Psychosomatics, Vol. 68, No. 3, 1999, pp. 114-150. doi: $10.1159 / 000012323$

[14] C. Kirschbaum and D. H. Hellhammer, "Salivary Cortisol in Psychoneuroendocrine Research: Recent Developments and Applications," Psychoneuroendocrinology, Vol. 19, No. 4, 1994, pp. 313-333. doi:10.1016/0306-4530(94)90013-2

[15] Y. Noto, T. Sato, M. Kudo, K. Kurata and K. Hirota, "The Relationship between Salivary Biomarkers and State-Trait Anxiety Inventory Score under Mental Arithmetic Stress: A Pilot Study," Anesthesia \& Analgesia, Vol. 101, No. 6, 2005, pp. 1873-1876. doi:10.1213/01.ANE.0000184196.60838.8D

[16] D. Kajdaniuk, B. Marek, E. Swietochowska, N. Ciesielska-Kopacz and B. Buntner, "Is Positive Correlation between Cortisol and Met-Enkephalin Concentration in Blood of Women with Breast Cancer a Reaction to Stress before Chemotherapy Administration?" Pathophysiology, Vol. 7, No. 1, 2000, pp. 47-51. doi:10.1016/S0928-4680(00)00028-6

[17] M. H. Lasikiewicz, H. Hendrickx, D. Talbot and L. Dye, "Exploration of Basal Diurnal Salivary Cortisol Profiles in Middle-Aged Adults: Associations with Sleep Quality and Metabolic Parameters," Psychoneuroendocrinology, Vol. 33, No. 2, 2008, pp. 143-151. doi:10.1016/j.psyneuen.2007.10.013

[18] J. M. Turner-Cobb, S. E. Sephton, C. Koopman, J. BlakeMortimer, D. Spiegel, "Social Support and Salivary Cortisol in Women with Metastatic Breast Cancer," Psychosomatic Medicine, Vol. 62, No. 3, 2000, pp. 337-345. 